УДК 534.1

\author{
Е.С. Лапшин ${ }^{1}$ Р.Н. Молчанов ${ }^{2}$, М.Ю. Милейковский ${ }^{3}$, Б.А. Блюсс ${ }^{1}$ \\ ${ }^{1}$ Институт геотехнической механики имени М. С. Полякова \\ НАН Украины, г. Днепр \\ ${ }^{2}$ Днепропетровская медииинская академия МОЗ Украинь \\ ${ }^{3}$ Киевский международный университет
}

\title{
ОБОБЩЕНИЕ ЧИСЛЕННОГО МОДЕЛИРОВАНИЯ НЕЛИНЕЙНЫХ КОЛЕБАНИЙ ПОЛУШАРА НА ПЛОСКОСТИ
}

\begin{abstract}
Рассмотрена задача о собственных нелинейных колебаниях абсолютно жесткого полушара на горизонтальной плоскости в предположении отсутствия диссипации энергии, проскальзывания и опрокидывания на основание полушара. Показано важное практическое значение задачи для расчета динамических гасителей низкочастотных колебаний (менее 10 Гц, а нередко и меньше 1 Гц) различных крупногабаритных сооружений: опоры ветроэлектрических установок, высотные здания, мосты и т.д. Рассмотрен один из простейших гасителей, который выполненный в виде полушара. Его колебания описываются нелинейным дифференциальным уравнением. Для настройки гасителя на частоту, близкую к частоте основного тона колебаний сооружения важно знать собственную частоту гасителя колебаний. Eе, как правило, определяют в предположении о малости амплитуды колебаний, что позволяет линеаризовать уравнение движения. Численные методы, которые применяются для решения нелинейных дифференциальных уравнений движения при больших амплитудах, позволяют найти только частные решения для конкретных условий. Возникает необходимость в обобщении частных решений. В работе выполнено сравнение собственной частоты линеаризованной системы и нелинейной системы. Последняя определялась с использованием метода Рунге-Кутта-Фальберга порядка 4-5. Рассчитана относительная погрешность вычисления собственной частоты, которая обусловлена линеаризацией (с увеличением амплитуды погрешность квадратично возрастает). Показано, что отношение собственной частоты линеаризованной системы к собственной частоте нелинейной системы не зависит от массы и радиуса полушара. Этот вывод сделал возможным обобщить результаты частных численных решений и получить формулу, которая учитывает влияние амплитуды на частоту собственных колебаний и позволяет по собственной частоте, определенной аналитически, для малых начальных углов (менее двадцати градусов) определить собственную частоту для начальных углов до девяноста градусов.
\end{abstract}

Ключевые слова: полушар, нелинейное дифференциальное уравнение, собственная частота. численное решение, аппроксимация, обобщение

Розглянуто задачу про власні нелінійних коливаннях абсолютно жорсткої напівкулі на горизонтальній площині в припущенні відсутності дисипації енергії, прослизання і перекидання на підставу напівкулі. Показано важливе практичне значення задачі для розрахунку динамічних гасителів низькочастотних коливань (менше 10 Гц, а нерідко і менше 1 Гц) різних великогабаритних споруд: опори вітроелектричних установок, висотні будівлі, мости і т. д. Розглянуто один 3 найпростіших гасителів, який виконаний у вигляді напівкулі. Його коливання описуються нелінійним диференціальним рівнянням. Для настройки гасителя на частоту, близьку до частоти основного тону коливань споруди, важливо знати власну частоту гасителя коливань. Ї̈̈, як правило, визначають в припущенні про малість амплітуди коливань, що дозволяс лінеаризовати рівняння руху. Чисельні методи, які застосовуються для вирішення нелінійних диференціальних рівнянь руху при великих амплітудах, дозволяють знайти лише частинні розв'язки за конкретних умов. Виникас необхідність в узагальненні частинних розв'язків. В роботі виконано порівняння власної частоти лінеаризованої системи і нелінійної системи. Остання визначалася з використанням методу Рунге-Кутта-Фальберга порядку 4-5. Розрахована відносна похибка обчислення власної частоти, яка обумовлена лінеаризацією (зі

(c) Лапшин Е.С., Молчанов Р.Н., Милейковский М.Ю., Блюсс Б.А., 2018 
збільшенням амплітуди похибка квадратично зростас). Показано, що відношення власної частоти лінеаризованої системи до власної частоти нелінійної системи не залежить від маси і радіусу напівкулі. Цей висновок зробив можливим узагальнити результати частинних чисельних розв'язків і отримати формулу, що враховус вплив амплітуди на частоту власних коливань і дозволяс по власній частоті, визначеній аналітично для малих початкових кутів (менше двадцяти градусів), визначити власну частоту для початкових кутів до дев'яноста градусів.

Ключові слова: напівкуля, нелінійне диференціальне рівняння, власна частота, чисельний розв'язок, апроксимація, узагальнення

The paper considers the problem of nonlinear oscillations of a completely solid hemisphere on a horizontal plane with dissipation being neglected, and in the absence of slipping or overturning on the base of the hemisphere. Practical importance of this problem lies in its applicatios to computing the details of dynamic dampers of low-frequency oscillations (with frequencies lower than $10 \mathrm{~Hz}$, sometimes even lower than $1 \mathrm{~Hz}$ ) of various bulky facilities, such as wind turbine towers, skyscrapers, bridges etc. We consider one of the simplest types of dampers in the shape of a hemisphere, whose oscillations are described by a nonlinear differential equation. In order to tune the damper to a frequency close to the main intrinsic frequency of oscillations of the facility, it is important to know intrinsic frequency of oscillation damper. This frequency is usually determined under assumption of small-amplitude oscillations, which allows one to linearise the associated equation of motion. Numerical methods that are used for solving nonlinear differential equations for larg amplitudes only yield solutions for particular choices of initial conditions. This necessitates the generalisation of these particular solutions. In this paper we have compared intrinsic frequencies of the linearised system with the frequency of the full nonlinear system that was computed numerically using the Runge-Kutta-Fehlberg method. We show that the ratio of the intrinsic frequency of the linearised system to the intrinsic frequency of the nonlinear system does not depend on the mass or radius of the hemisphere. This conclusion allowed us to generalise the results of particular numerical soultions and derive an expression for the intrinsic frequency of nonlinear oscillations with initial angles up to $90^{\circ}$. This expression takes into account the effects of the amplitude of oscillations, and is expressed through an analytically derived intrinsic frequency of oscillations for small initial angles (less than 20 degrees).

Key words: hemisphere, nonlinear differential equation, natural frequency. numerical solution, approximation, generalization

Введение. Динамические гасители колебаний широко используются для снижения нагрузок в различных механизмах и инженерных сооружениях [1]. Для крупногабаритных сооружений: опоры ветроэлектрических установок, высотные здания, мосты и т.д. - характерны низкочастотные собственные колебания (менее 10 Гц, а нередко и меньше 1 Гц [2, 3]). В этом случае применяют гасители колебаний с телами качения [2-6], которые имеют простую конструкцию и высокую надежность. Их обзор приведен в работах $[2,6]$. Гаситель настраивают на частоту, близкую к частоте основного тона колебаний сооружения [2]. Для этого важно знать собственную частоту гасителя колебаний. Ее, как правило, определяют в предположении о малости амплитуды колебаний, что позволяет линеаризовать уравнение движения. При больших амплитудах нелинейное дифференциальное уравнение движения решают численными методами, которые, однако, позволяют найти только частные решения для конкретных условий. Возникает необходимость в обобщении частных решений.

Цель работы - определить параметры динамического гасителя колебаний с телом качения, обеспечивающие требуемую собственную частоту нелинейных колебаний и обобщение численных результатов.

Постановка задачи. Рассмотрим один из простейших гасителей [6], выполненный в виде полушара 1, совершающего колебания на плоскости 2 (рис. 1). При решении ряда прикладных задач, связанных с вибрационным воздействия на сыпучую среду $[4,5]$, колебания полушара могут служить в качестве модельного представления движения твердых частиц.

104 


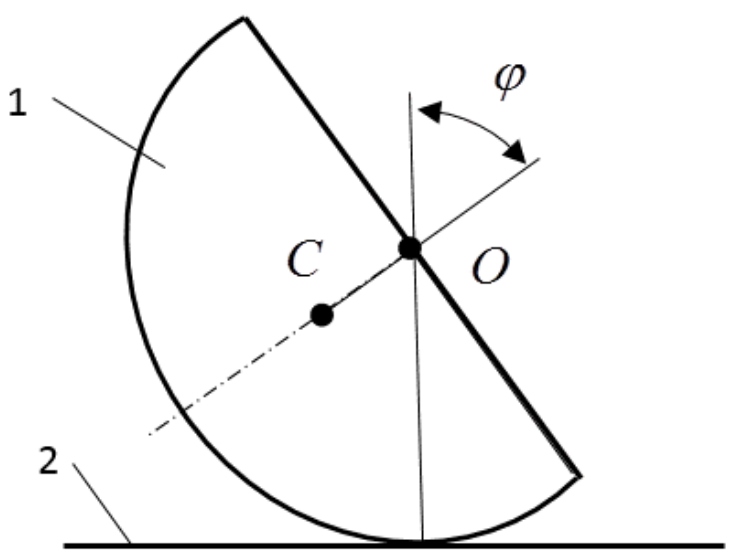

Рис. 1. Расчетная схема гасителя колебаний: 1 - полушар; 2 - плоскость

При качании полушара предположим, что отсутствуют диссипация энергии, проскальзывание и опрокидывание на основание.

Уравнение колебаний полушара $[6,9,10]$ :

$$
I_{p} \ddot{\varphi}+m l^{2} \sin \varphi \cos \varphi \cdot \dot{\varphi}^{2}+m g l \sin \varphi=0,
$$

где $I_{p}$ - момент инерции полушара относительно мгновенного центра скоростей; $\varphi$ - угол; $m$ - масса полушара; $l$ - расстояние центра масс $C$ от основания $O$ полушара; $g$ - ускорение свободного падения.

Точка над буквами означает дифференцирование по времени. Начальные условия: $\varphi=\varphi_{0}$ и $\dot{\varphi}=0$. Поскольку диссипация не учитывается, то $\varphi_{0}-$ амплитуда собственных колебаний.

Момент инерции $I_{p}$ и расстояние $l$ вычисляются по формулам:

$$
I_{p}=m l^{2}\left(\frac{83}{45}+\sin ^{2} \varphi\right), l=\frac{3}{8} r,
$$

где $r$ - радиус основания полушара.

Собственная частота линеаризованной системы. Нелинейное уравнение (1) не имеет аналитического решения. Для его линеаризации предполагают, что угол $\varphi$ мал и принимают $\sin \varphi \approx \varphi, \cos \varphi \approx 1$.

В результате получают

$$
I_{c} \ddot{\varphi}+m g l \varphi=0,
$$

где $I_{c}=\frac{83}{320} m r^{2}$ - момент инерции полушара относительно горизонтальной оси, проходящей через центр инерции и перпендикулярной к плоскости чертежа.

Разделив (2) на $I_{c}$ получим

$$
\ddot{\varphi}+\omega_{0}^{2} \varphi=0 .
$$

где $\omega_{0}=\sqrt{\frac{120 g}{83 r}}-$ собственная круговая частота. 
Тогда частота собственных колебаний

$$
v_{0}=\frac{1}{2 \pi} \sqrt{\frac{120 g}{83 r}} .
$$

Собственная частота нелинейной системы. Для определения собственной частоты нелинейных колебаний можно для каждого конкретного значения $r$ и $\varphi_{0}$ численно решать уравнение (1), однако, это делает трудоемким обобщение результатов. Задача упрощается, если учесть, что на основании теоремы об изменении кинетической энергии, получена угловая скорость [10]

$$
\dot{\varphi}=4 \cdot \sqrt{\frac{g\left(\cos \varphi-\cos \varphi_{0}\right)}{3 r\left(83 / 45+\sin ^{2} \varphi\right)}} .
$$

Полушар поворачивается на угол $d \varphi$ за время

$$
d t=\frac{d \varphi}{\dot{\varphi}} .
$$

Интегрируя обе части уравнения (4), определим период $T$ колебаний и собственную частоту $v$ с учетом нелинейности

$$
T=\int_{0}^{\varphi_{0}} \sqrt{\frac{3 r\left(83 / 45+\sin ^{2} \varphi\right)}{g\left(\cos \varphi-\cos \varphi_{0}\right)}} d \varphi, \quad v=\frac{1}{T} .
$$

При интегрировании учтена симметрия фазового портрета относительно осей координат, что позволило задать пределы 0 и $\varphi_{0}$, а для получения периода результат увеличить в четыре раза. На рис. 2 приведен характерный вид фазового портрета, который получен численным интегрированием уравнения (1) методом Рунге-Кутта-Фальберга порядка 4-5 при $r=0,1 \mathrm{M}, \varphi_{0}=5^{\circ}, 10^{\circ}, 30^{\circ}, 60^{\circ}, 90^{\circ}$.

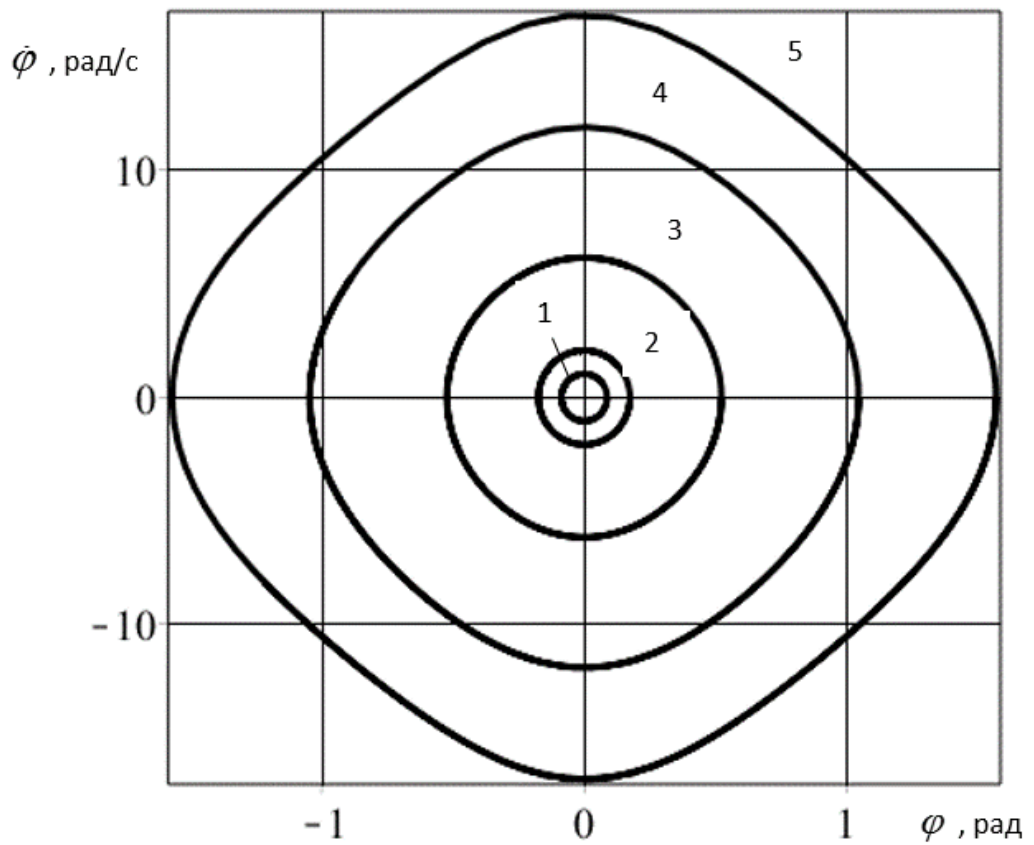

Рис. 2. Характерный вид фазового портрета колебаний полушара:

$$
\left(r=0,1 \text { м, где } 1,2,3,4 \text { и } 5-\varphi_{0}=5^{\circ}, 10^{\circ}, 30^{\circ}, 60^{\circ}, 90^{\circ}\right)
$$


Здесь и далее вычисления выполнены в математическом пакете Maple.

Обобщение частных численных решений. Найдем отношение собственных частот

$$
v^{*}=\frac{v_{0}}{v}=\frac{3}{\pi} \sqrt{\frac{10}{83}} \int_{0}^{\varphi_{0}} \sqrt{\frac{83 / 45+\sin ^{2} \varphi}{\cos \varphi-\cos \varphi_{0}}} d \varphi .
$$

Эту величину назовем относительной собственной частотой.

Акцентируем внимание на том, что $v *$ не зависит от массы и размеров полушара.

Относительная погрешность, обусловленная линеаризацией уравнения (1)

$$
\delta=\frac{v_{0}-v}{v_{0}} \cdot 100 \%=\left(1-v^{*}\right) \cdot 100 \% .
$$

На рис. 3 круглыми маркерами представлены результаты численного интегрирования методом Ньютона-Котеса выражения (6), а треугольными погрешности.

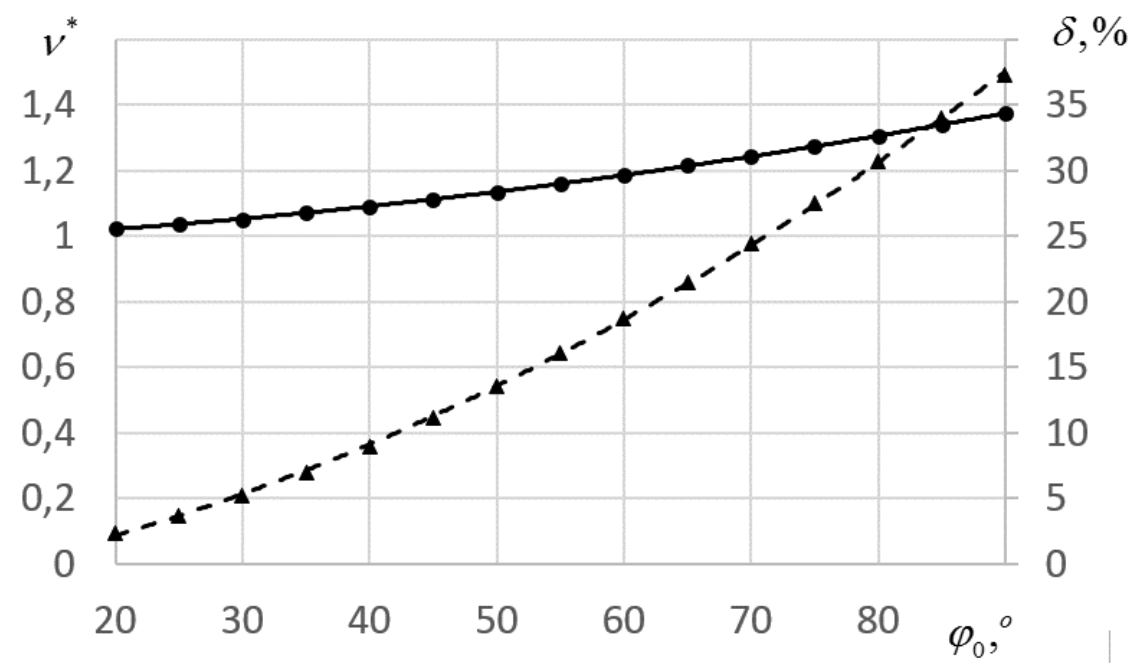

Рис. 3. Зависимость относительной собственной частоты $v *$ и погрешности $\delta$ от начального угла $\varphi_{0}$ :

сплошная линия - зависимость $\nu *-\varphi_{0}$;

пунктирная линия - зависимость $\delta-\varphi_{0}$

При амплитудах больше 60 градусов погрешность превышает 20 \%. Линиями изображены результаты аппроксимации функциями $\left(20^{\circ} \leq \varphi_{0} \leq 90^{\circ}\right)$ :

$$
\begin{gathered}
v^{*}=3 \cdot 10^{-5} \varphi_{0}^{2}+1,6 \cdot 10^{-3} \varphi_{0}+0,9763, \\
\delta=3,1 \cdot 10^{-3} \varphi_{0}^{2}+0,1621 \varphi_{0}-2,3747 .
\end{gathered}
$$

Аппроксимация выполнена в интервале $20^{\circ} \leq \varphi_{0} \leq 90^{\circ}$ методом наименьших квадратов и обеспечивает высокую точность (коэффициент детерминации 0,999 ).

Если заданы радиус и начальное отклонения, то формулы (7) и (8) позволяют определить собственную частоту колебаний полушара и погрешность при линеаризации уравнения колебаний. Поскольку, как показано выше $v^{*}$ не зависит 
от массы и размеров полушара, учитывая (6) и (7), получим выражение для определения собственной частоты нелинейных колебаний

$$
v=v_{0}\left(3 \cdot 10^{-5} \varphi_{0}^{2}+1,6 \cdot 10^{-3} \varphi_{0}+0,9763\right)^{-1} .
$$

Формула (9) обобщает результаты численных экспериментов, направленных на исследование влияния начального угла $\varphi_{0}$ на собственную частоту колебаний полушара. Она позволяет по аналитически определяемой собственной частоте $v_{0}$ для малых начальных улов $\left(\varphi_{0} \leq 20^{\circ}\right)$ определить частоту $v$ для начальных углов до $90^{\circ}$.

Выводы. Получены формулы (3), (5), определяющие влияние радиуса $r$ полушара на собственные частоты $v_{0}$ и $v$ как при малых, так и больших амплитудах $\varphi_{0}$ колебания. В первом случае колебания линейные, а во втором нелинейные, зависящие от амплитуды.

Показано, отношение частот $v *=v_{0} / v$ не зависит от радиуса полушара. Это позволило обобщить результаты численных экспериментов и установить - влияние амплитуды на относительную частоту описывается полиномом второй степени (7).

Определена относительная погрешность (8) вычисления собственной частоты, обусловленная линеаризацией.

\section{Библиографические ссылки}

1. Вибрации в технике: Справочник. В 6-ти т. Т. 6. Защита от вибрации и ударов [Текст] / Под ред. К. В. Фролова.- М.: Машиностроение, 1981. -/1981. - 456 с.

2. Legeza, V.P. Dynamics of vibroprotective systems with roller dampers of low-frequency vibrations [Text] / V.P. Legeza // Strength of Materials. - 2004. - №2 (36). - P. 186-194.

3. Takei, H. Vibration control effects of tuned cradle damped mass damper [Text] / H. Takei, Y. Shimazaki // Journal of Applied Mechanics. - 2010. - № 13. - P. 587-594.

4. Naruse, T. Entwicklung eines passiven dynamischen Schwingungsdämpfers [Text] / T. Naruse, Y. Hirashima // Stahlbau. - 1987. - №7 (56). - S. 193-196.

5. Pirner, M. Dissipation of kinetic energy of large-span bridges [Text] / M. Pirner // Acta Technica CSAV. - 1994. - № 39. - P. 645-655.

6. Bransch, M. Unbalanced oil filled sphere as rolling pendulum on a flat surface to damp horizontal structural vibrations / M. Bransch // Journal of Sound and Vibration. - 2016. - № 368. P. 22-35.

7. Надутый, В.П. Вероятностные процессы вибрационной классификации минерального сырья [Текст] / В.П. Надутый, Е.С. Лапшин. - К.: «Наукова думка», 2005. $180 \mathrm{c}$.

8. Лапшин, Е.С. Вероятностная оценка скорости вибротранспортирования слоя сыпучего материала / Е.С. Лапшин // Вибрации в технике и технологиях: Всеукр. науч.техн. журн. - Винница, 2004. - № 3(35). - С. 64-67.

9. Маркеев, А.П. Динамика тела, соприкасающегося с твердой поверхностью [Текст]/ А.П. Маркеев. - М.: Наука, Гл. ред. физ.-мат. лит., 1992. - 336 с.

10.Теоретическая механика в примерах и задачах, т. 3 (специальные главы механики) [Текст] / М.И. Бать, Г.Ю. Джанелидзе, А.С. Кельзон, под редакцией Г.Ю. Джанелидзе. - М.: «Наука», 1973. - 488 с.

Надійшла до редколегії 25.04.2018

108 